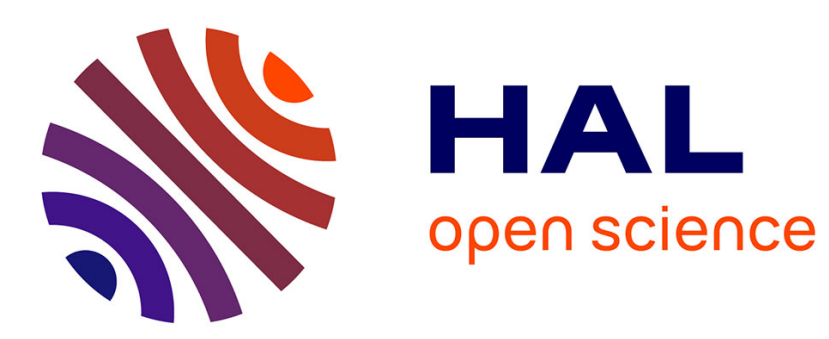

\title{
La critique de la théorie des ensembles dans la dissertation de Brouwer (1907)
}

Michel Bourdeau

\section{To cite this version:}

Michel Bourdeau. La critique de la théorie des ensembles dans la dissertation de Brouwer (1907).

Mathématiques et Sciences Humaines, 2003, 164, 10.4000/msh.2892 . halshs-01633692

\section{HAL Id: halshs-01633692 \\ https://shs.hal.science/halshs-01633692}

Submitted on 13 Nov 2017

HAL is a multi-disciplinary open access archive for the deposit and dissemination of scientific research documents, whether they are published or not. The documents may come from teaching and research institutions in France or abroad, or from public or private research centers.
L'archive ouverte pluridisciplinaire $\mathbf{H A L}$, est destinée au dépôt et à la diffusion de documents scientifiques de niveau recherche, publiés ou non, émanant des établissements d'enseignement et de recherche français ou étrangers, des laboratoires publics ou privés. 


\section{msh-mss $\begin{array}{ll}\text { Mathématiques } \\ \text { et sciences humaines }\end{array}$}

Mathematics and social sciences

164 | Hiver 2003

Varia

\section{La critique de la théorie des ensembles dans la dissertation de Brouwer (1907)}

The criticism of set theory in Brouwer's dissertation (1907)

\section{Michel Bourdeau}

revues.org

Édition électronique

URL : http://msh.revues.org/2892

DOI : $10.4000 /$ msh.2892

ISSN : 1950-6821
Éditeur

Centre d'analyse et de mathématique sociales de l'EHESS

Édition imprimée

Date de publication : 1 décembre 2003

ISSN : 0987-6936

Ce document vous est offert par SCD de

I'Université Paris 1 Panthéon-Sorbonne

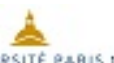

PANTHÉON SORBONNE

Référence électronique

Michel Bourdeau, "La critique de la théorie des ensembles dans la dissertation de Brouwer (1907) », Mathématiques et sciences humaines [En ligne], 164 | Hiver 2003, mis en ligne le 18 février 2006 , consulté le 13 novembre 2017. URL : http://msh.revues.org/2892 ; DOI : 10.4000/msh.2892 


\title{
LA CRITIQUE DE LA THEORIE DES ENSEMBLES DANS LA DISSERTATION DE BROUWER $(1907)^{1}$
}

\author{
Michel BOURDEAU ${ }^{2}$
}

\begin{abstract}
RESUME - S'il faudra attendre 1917 pour que Brouwer développe une mathématique distincte des mathématiques classiques, certains des thèmes caractéristiques de l'intuitionnisme, comme l'attachement à une intuition de type kantien ou l'idée que le continu est une donnée irréductible, apparaissent dès la Dissertation de 1907. C'est le cas en particulier de l'attitude à l'égard de la création cantorienne, où il convient, nous dit-on, de distinguer deux aspects : les acquis proprement mathématiques (topologie, ordinaux), qu'il s'agit de sauvegarder, une confiance excessive dans les pouvoirs de la logique, qui est responsable des contradictions. Le transfini se présente ainsi sous deux formes : la théorie des puissances, les ordinaux. Brouwer accepte celle-ci mais non celle-là. La même attitude explique encore que l'hypothèse du continu soit examinée en deux endroits différents : dans la première partie, pour sa version proprement mathématique, dans la troisième, pour sa version logique. Dans ce dernier cas, Brouwer admet les deux principes de construction des ordinaux, mais estime que cela n'autorise pas à considérer la seconde classe de nombres comme une totalité achevée.
\end{abstract}

MOTS-CLES - Intuitionnisme, Transfini, Continu, Ordinaux

SUMMARY - The criticism of Set Theory in Brouwer's Dissertation (1907)

Although Brouwer had not developed intuitionistic mathematics by 1917, some of his main ideas are already to be found in his 1907 Dissertation, for instance his attitude towards set theory. We are invited to distinguish two aspects in Cantor's creation: the authentic mathematical contribution (topology, ordinals), and a spurious one, which leads to contradictions and reflects an excessive confidence in the power of logic. Consequently, the transfinite has two faces: the hierarchy of the alephs, and the ordinals. Brouwer agrees with the latter, not with the former. This explains also why the Continuum Hypothesis is discussed in two different places: the mathematical version in the first chapter, the logical one in the third chapter. In the latter, Brouwer accepts the two generating principles for ordinals, but he thinks that these provide no ground for taking the second class of numbers as a completed totality.

KEYWORDS - Intuitionism, Transfinite, Continuum, Ordinals

La Dissertation. 1907-1908 marquent des années fastes dans les annales des mathématiques, puisque c'est dans ce court laps de temps que furent formulées les trois grandes parades à la crise des fondements : la théorie des types, l'axiomatisation de Zermelo, et la nouvelle théorie des ensembles conçue par Brouwer. Des trois textes publiés alors, celui du mathématicien hollandais n'est pas seulement le plus ancien,

\footnotetext{
${ }^{1}$ 'Article reçu le 20 janvier 2003, révisé le 10 juin 2003, accepté le 13 octobre 2003.

${ }^{2}$ Centre d'analyse et de mathématique sociales, École des hautes études en sciences sociales, 54 bd Raspail 75270 Paris cedex 06, e-mail!: bourdeau@ehess.fr
} 
puisqu'il précède les deux autres d'une année, il est aussi certainement le moins lu. Diverses raisons expliquent cette relative désaffection : tout d'abord, la reconstruction annoncée des mathématiques devait passer, on le sait aujourd'hui, par la découverte des suites de choix du point de vue qui nous occupe, c'est donc dans des textes postérieurs à 1917 qu'il faut chercher la nouvelle théorie des ensembles et du continu qui constitue le coeur de l'intuitionnisme. Des travaux antérieurs à 1917, on retient plutôt ce qui touche à la topologie, ou des articles accessibles dans une langue autre que le hollandais, comme un article sur la théorie des puissances, ou la leçon inaugurale de 1912, Intuitionnisme et formalisme, traduite dès l'année suivante en anglais. Si donc la dissertation préparée sous la direction de Korteweg et présentée le 19 février 1907 par le jeune mathématicien n'a guère été étudiée $e^{3}$, elle n'en constitue pas moins incontestablement une œuvre de percée et il serait très regrettable d'en sous-estimer la portée : l'auteur n'affirmait-il pas avoir résolu pas moins de trois des problèmes figurant sur la fameuse liste dressée quelques années plus tôt par Hilbert, à commencer par le premier, concernant l'hypothèse du continu ?

Il suffit de parcourir l'ouvrage pour mesurer l'originalité du point de vue adopté : l'auteur s'était fait remarquer deux ans plus tôt par un opuscule intitulé Vie, art et mysticisme; il n'y a donc pas à s'étonner s'il n'hésite pas, pour justifier ses positions, à s'engager sur le terrain de la philosophie. Les démonstrations y alternent avec des considérations sur les rapports des mathématiques au langage, ou à l'expérience. Korteweg n'avait donc pas tort, qui retournait à son étudiant une première version de la thèse, accompagnée des commentaires suivants :

\begin{abstract}
Honnêtement, Brouwer, je ne peux pas l'accepter dans sa forme actuelle. [...] S'y mêle partout une sorte de pessimisme et d'attitude mystique devant la vie qui n'est pas de la mathématique et qui n'a rien à voir avec la mathématique. [...] Quelqu'un pourrait être en total désaccord avec vous sur ces sujets, et partager vos idées sur les fondements des mathématiques ${ }^{4}$.
\end{abstract}

Le rapport à Cantor. Du texte de 1907, un seul aspect sera examiné ici : le rapport à Cantor. Non seulement, celui-ci est clairement fixé dès cette époque, pour ne plus varier par la suite, mais surtout, il importe de souligner l'importance de la création cantorienne pour Brouwer. Le plus souvent, on ne retient que la condamnation!; s'en tenir là serait cependant tout à fait unilatéral. Dans la théorie des ensembles, il n'y a pas que des erreurs, il y a aussi de nouveaux domaines pour les mathématiques, dont il serait regrettable de se passer. Ce que je cherche à souligner ici, c'est une idée tout à fait simple, triviale, mais qui, précisément pour cette raison, risque d'être négligée : dire que l'intuitionnisme est une réaction au cantorisme, c'est admettre que celui-ci a été tout à fait déterminant pour Brouwer, que l'œuvre de ce dernier est impensable sans référence aux découvertes du mathématicien allemand; et, dans la mesure où ce qui est proposé n'est nullement un retour au statu quo, cela revient à reconnaître que, dans la création

\footnotetext{
${ }^{3}$ Voir cependant!: W. van Stigt, Brouwer's Intuitionism, Amsterdam, North Holland, 1990, et D. van Dalen, Mystic, Geometer and Intuitionist, The Life of L. E. J. Brouwer, vol. 1, The Dawning Revolution, Oxford, Clarendon Press, 1999, p. 80-122.

D. van Dalen a donné une réédition annotée de la Dissertation (L. E. J. Brouwer en de Gronslagen van de Wiskunde, Utrecht, Epsilon Uitgaven, 2001) que mon ignorance du néerlandais ne m'a malheureusement pas permis d'utiliser ; toutes les références renvoient à la traduction anglaise publiée au tome 1 des Collected Works, édité par A. Heyting, Amsterdam, North Holland, 1975.

${ }^{4}$ Cité par W. van Stigt dans l'introduction de sa traduction anglaise, Life, Art, and Mysticism, de l'ouvrage de 1905, Notre Dame Journal of Formal Logic 37, 1996, p. 383.
} 
cantorienne, tout n'est pas à rejeter, ou encore, que les contributions positives de Cantor aux mathématiques ne sont nullement négligeables. Cette attitude à la fois réceptive et critique n'est pas propre au fondateur de l'intuitionnisme. Un peu plus tôt, c'était la position de Borel et de ceux qu'on appellera les pré-intuitionnistes ${ }^{5}$. Comprendre la position de Brouwer à l'égard de la théorie des ensembles, c'est donc commencer par distinguer ce qu'il en accepte et ce qu'il n'en accepte pas.

S'il faut ainsi commencer par séparer le bon grain de l'ivraie, c'est que, aux yeux de Brouwer, l'œuvre de Cantor a pour originalité de présenter deux aspects : l'un mathématique, l'autre logique. Ce que voulait Brouwer, c'est sauver le contenu proprement mathématique de la théorie des ensembles. Plus précisément encore, pour comprendre comment il aborde la théorie des ensembles, ce qu'il en attend, il convient de ne jamais perdre de vue que les mathématiques intuitionnistes visent avant tout à donner une bonne théorie du continu et des réels. Pas un instant, donc, Brouwer n'a été tenté par l'idée d'abolir la théorie des ensembles, au profit par exemple d'une méréologie. De façon un peu provocatrice, on peut résumer en ces termes le but qu'il s'était proposé : constituer une nouvelle théorie des ensembles ${ }^{6}$. D'où les équivoques bien connues de son vocabulaire, où ensemble (Menge) sert à désigner ce que nous appelons aujourd'hui déploiement; d'où encore les incompréhensions qui en ont résulté et qui ont lourdement grevé la diffusion initiale de ses idées. Il est même possible de préciser ce contenu purement mathématique : c'est celui qui se situe dans le prolongement de l'analyse, de la théorie des fonctions, de la topologie, dont on sait historiquement qu'elles ont effectivement constitué le point de départ de la réflexion de Cantor. L'ivraie, dira-t-on, c'est l'infini actuel. Brouwer préfère employer un autre langage : ce qu'il faut avant tout reprocher à la création cantorienne, c'est d'avoir laissé la logique contaminer les mathématiques. La logique n'est pas seulement stérile; elle est d'abord et avant tout totalement étrangère à l'univers mathématique. Cantor a été ainsi conduit à substituer au raisonnement mathématique, dans lequel la pensée construit son objet, un verbalisme creux, tout au plus adapté au traitement des domaines finis. Si l'on veut être un peu plus généreux à l'égard de la discipline créée par Aristote, l'on y verra une science empirique, dont les lois ne font que codifier les raisonnements tenus par les mathématiciens, et qui dépendent des domaines étudiés. La grandeur de Cantor, c'est de nous avoir ouvert l'accès à de nouveaux domaines, et son erreur, c'est d'avoir cru qu'il pouvait y appliquer sans examen les modes de raisonnement qui avaient fait leur preuve dans les domaines usuels, c'est-à-dire en l'occurrence finis.

L'existence de cette double attitude est reflétée dans l'organisation du texte, qui aborde le sujet en deux endroits. La question du continu, c'est-à-dire l'aspect proprement mathématique, est examinée dans la première partie, intitulée la construction des mathématiques, qui consacre une section aux divers ensembles-depoints possibles (p. 44-47), tandis que la troisième et dernière partie, mathématique et logique, contient une section intitulée rejet des fondements logiques de la théorie des ensembles (p. 80-87). Tout ce qui touche à la seconde classe de nombres en relève. La

\footnotetext{
${ }^{5}$ Cf. H. Gispert, «!La théorie des ensembles en France avant la crise de 1905 : Baire, Borel, Lebesgue... et tous les autres!», Revue d'histoire des mathématiques, t. 1, 1995, p. 28-42.

${ }^{6}$ Comme le remarque Dummett, l'exigence d'effectivité, par exemple dans le cas de la théorie des réels récursifs, «!a conduit au rejet global de l'un des composants fondamentaux du concept classique. L'intuitionnisme, cependant, vise à réformer les mathématiques, non à les émonder ; de son point de vue, à peu près aucune des idées des mathématiques classiques n'est tout à fait fallacieuse mais, ayant été systématiquement mal construites, elles sont toutes déformées!» (Elements of Intuitionism, 2d Edition, Oxford, Oxford Logic Guides 39, Clarendon Press, 2000, p. 45).
} 
dualité de point de vue indiquée à l'instant se retrouve donc à l'intérieur de la théorie cantorienne du continu, ce qui confirme la place centrale que ce concept occupe dès le début chez Brouwer. La seconde classe de nombres n'existe pas et, alors même que la théorie axiomatique des ensembles est encore à naître, l'erreur de Cantor sur ce point est attribuée à ce qu'il partage le même point de vue que les axiomaticiens. Tout ce qui, dans la théorie du continu, fait intervenir la seconde classe de nombres doit donc être rejeté hors des mathématiques et tenu pour plus ou moins privé de sens.

Limites du point de vue adopté. S'en tenir à 1907, comme ce sera ici le cas - sans se priver toutefois de renvoyer le cas échéant à quelques autres textes antérieurs à 1917, comme la leçon inaugurale de 1912, qui met en évidence le rapport entre logique et mathématique implicite dans l'axiome de compréhension entendu comme mode de formation d'ensembles - ; s'en tenir à 1907, donc, c'est renoncer à présenter l'œuvre de Brouwer dans ce qu'elle a de plus original ${ }^{7}$ : avec l'intuitionnisme, on a bien affaire à une autre mathématique, où l'on démontre des théorèmes qui ne sont pas classiquement vrais, comme par exemple que toute fonction définie sur l'intervalle [0,1] est continûment uniforme. Afin d'éviter tout malentendu, il importe de souligner qu'il n'y a pas de «!bonne!» théorie intuitionniste du continu avant 1917, i.e. avant la découverte des suites de choix : elles permettent en effet de combler la distance séparant le continu réduit et le continu authentique puisqu'aux suites engendrées par une loi définie à l'avance, les seules disponibles jusqu'alors, viennent maintenant s'ajouter des suites arbitraires obtenues par des choix arbitraires effectués successivement dans le temps ${ }^{8}$. Le fait permet de mesurer plus précisément la portée de la dissertation, en la situant dans l'ensemble de l'œuvre de Brouwer. Le contenu en sera en partie récusé comme insatisfaisant. La présenter comme une œuvre de percée, c'est admettre qu'il s'agit aussi d'une œuvre de transition, qui contient des tentatives prématurées, des branches qu'il faudra couper. D'où le statut équivoque d'une œuvre où la pensée de l'auteur n'est pas encore parvenue à sa pleine maturité : l'ouvrage contient mainte obscurité, ce qui autorise rétrospectivement à adresser à 1907 le même reproche que celui-ci adresse à Cantor : d'être parfois peu intelligible, de ne pas toujours faire sens.

En première approximation, on serait tenté de dire que l'apport de l'ouvrage se limite à l'analyse critique. 1917 marque une coupure : avant, on s'en tient à la critique, il s'agit de faire table rase ; après, c'est la période créatrice, la reconstruction des mathématiques sur une base nouvelle enfin découverte. Cette description s'avère toutefois un peu inexacte. Certes, la partie critique prédomine, en ce sens que la bonne théorie ne viendra que plus tard ; mais dès cette date, des principes sont posés, qui ne seront pas remis en cause : que les jugements mathématiques sont synthétiques en ce sens qu'ils reposent sur une intuition primordiale ; que le langage, et partant la logique, sont étrangers à la mathématique ; la validité de la théorie des ordinaux, etc.

\footnotetext{
${ }^{7}$ A fortiori, c'est renoncer à parler de tout ce qui s'est passé hors de l'intuitionnisme depuis cette date. Cent ans après la conférence de Hilbert, L'hypothèse du continu reste un des principaux problèmes ouverts des mathématiques, sur laquelle se sont exercés les plus grands esprits. Sur les résultats obtenus depuis Cohen dans le cadre de ce qu'on a appelé parfois «!le programme de Gödel!», voir par exemple l'article de W. Hugh Woodin, The Continuum Hypothesis, Parts I and II, Notices of the AMS, 48, 2001, $\mathrm{n}^{\circ} 6$ et 7, p. 567-576 et 681-690. Sur la théorie intuitionniste du continu, on pourra se reporter à la conférence prononcée en 1928 et publiée en 1930, la structure du continu, traduite dans J. Largeault, Intuitionnisme et théorie de la démonstration, Vrin, 1992, p. 271.

${ }^{8}$ Cf. par exemple A. Troelstra et D. van Dalen, Constructivism in Mathematics, Amsterdam, North Holland, t. 2, 1988, p. 640.
} 
Pour effectuer ce double partage, l'un, synchronique, entre ce que, en 1907, Brouwer accepte et n'accepte pas de la théorie des ensembles ; 1'autre, diachronique, entre ce qui sera conservé et ce qui ne sera pas conservé par Brouwer de sa dissertation, - je procéderai en deux temps, examinant tout à tour ce qui touche à l'hypothèse du continu puis les principes illicites qui entrent en jeu dans la formation de la seconde classe de nombres et qui étendent leur effet jusque sur la question du continu. Dans les deux cas il faudra à nouveau distinguer deux temps et, avant d'exposer les positions brouweriennes, commencer par rappeler les positions de Cantor qui y sont discutées.

\section{L'HYPOTHESE DU CONTINU EN 1907}

Questions de méthode : problème du continu et hypothèse du continu. Géomètre, topologiste, l'intérêt de Brouwer pour la théorie des ensembles trouve sa source dans la question du continu et c'est par là qu'il convient de commencer. Et avant tout, il convient de bien voir quelle est au juste la question. Contrairement à ce qu'on pourrait être porté à croire, cela ne va en effet pas du tout de soi. Non seulement, il y a plusieurs façons de poser la question, qui ne sont pas toujours équivalentes ; mais, à en croire Brouwer, et quelques autres, il n'est pas sûr que ce soit toujours une question bien posée. Pour mesurer la place éminente qu'occupe l'hypothèse du continu dans les mathématiques de ce siècle, il suffit de rappeler que c'est par là que commençait la fameuse liste des problèmes dressée par Hilbert en 1900 : donner une démonstration de l'hypothèse du continu ; mais l'hypothèse du continu n'étant à son tour qu'une réponse au problème du continu, il convient tout d'abord de dire quelques mots de celui-ci.

Gödel y a consacré une brève monographie à juste titre célèbre : Qu'est ce que le problème du continu de Cantor ? ${ }^{9}$ Les premières lignes en donnent deux formulations : combien y a-t-il de points sur une ligne droite dans l'espace euclidien ? combien y a-t-il d'ensembles distincts d'entiers ? S'il est vrai que ces deux questions sont équivalentes, en ce sens qu'elles concernent l'une le continu géométrique, l'autre le continu arithmétique, la droite des réels, la seconde a toutefois ceci en propre d'être la seule à faire explicitement mention du concept d'ensemble. Ce qu'elles ont d'abord en commun, c'est de porter sur ce qu'Aristote appelait la catégorie de la quantité : combien...? Nous sommes invités à compter, ce qui relève de l'arithmétique cardinale et c'est pourquoi Gödel enchaîne aussitôt en exposant la théorie des cardinaux ou des puissances. Ce concept une fois dégagé, la question admet une troisième formulation : quelle est la puissance du continu ? Le combien ? initial se traduisant cette fois : lequel d'entre les alephs ? Une fois construite l'échelle des alephs, la question se pose en effet de savoir si elle est complète ; plus précisément encore : y a-t-il un nombre cardinal compris entre $\square_{0}$ et $2^{\square 0}$ ? où placer $2^{\square 0}$ dans l'échelle des alephs ? ${ }^{10}$

Ce qu'on appelle hypothèse du continu n'est autre que la réponse, négative, apportée par Cantor à cette dernière question!: c, le cardinal du continu, est le premier cardinal plus grand qu' $\square_{0}$, soit $\mathbf{c}=2^{\square 0}=\square_{1}$; c'est-à-dire encore : de deux choses l'une,

\footnotetext{
${ }^{9}$ K. Gödel, Collected Works, Oxford, Oxford U. P., t. 2 ; 1995, traduction française dans Largeault 1992, p. 509-531.

${ }^{10}$ Sur la question, cf. par exemple S. Feferman, In the Light of Logic, Oxford, Oxford University Press, 1998, p. 34-37.
} 
tout sous-ensemble infini du continu a soit la puissance de l'ensemble des nombres naturels, soit la puissance du continu tout entier.

On ne remarque peut-être pas assez à quel point l'approche de Cantor s'écarte de celle qui avait prévalu jusqu'alors. Il est permis en effet de penser que ce que l'on est tenté d'entendre tout d'abord par problème du continu n'a pas grand-chose à voir avec la théorie des cardinaux transfinis découverte par Cantor. Le continu fait problème depuis Zénon, et s'est ainsi constitué au cours des siècles un corps de doctrines, ou à tout le moins de concepts : le continu par exemple demande à être pensé en rapport avec la continuité, dans son opposition au discret, etc. ${ }^{11}$ Or tout cela disparaît, ou du moins passe au second plan, avec Cantor. Le problème du continu de Cantor n'a de sens que dans le cadre de la théorie des ensembles, ce qui n'est pas le cas du problème classique du continu. On peut dire que la théorie des ensembles permet de reformuler et de mieux poser le problème classique ; mais il est également permis de soutenir, avec Brouwer, qu'avec elle, «!le continu apparaissait dans une forme qui dépend des opérations logiques, donc entièrement différente de celle donnée par intuition pure a priori!» ${ }^{12}$. Hilbert n'était pas loin de donner raison à Brouwer quand il décrivait dans les termes suivants le problème du continu :

Lorsque Cantor eut découvert ses premiers nombres transfinis, les nombres
de la seconde classe, comme on les appelle, se posa la question de savoir si
l'on peut, au moyen de cette énumération transfinie, dénombrer réellement
les éléments d'ensembles rencontrés dans d'autres contextes et non
dénombrables au sens ordinaire. Le segment de droite vint au premier rang
des ensembles à considérer. La question de savoir si les points du segment de
droite, c'est-à-dire les nombres réels, peuvent être énumérés au moyen des
nombres de la seconde classe, est le fameux problème du continu, formulé
mais laissé ouvert par Cantor ${ }^{13}$.

De la même façon, l'expression hypothèse du continu est doublement malencontreuse : et sur hypothèse, puisqu'il faudrait plutôt dire conjecture et que depuis Hilbert, cette conjecture figure sur une liste de problèmes; et sur continu, puisqu'il s'agit en fait de la suite des alephs. Le problème du continu - c'est-à-dire non le problème du continu de Cantor, comme prenait bien soin d'ajouter Gödel, mais le problème classique du continu - a peut-être servi de point de départ à l'hypothèse du continu de Cantor, mais ce point de départ a vite été perdu de vue. Rien n'est plus clair à cet égard que l'hypothèse du continu généralisée, où il s'agit de savoir si l'équation $\square_{\mathrm{Q}+1}=2^{\mathrm{Qu}}$ est valide. Déjà on peut commencer à comprendre les réticences de certains

\footnotetext{
${ }^{11}$ Voir encore par exemple le chapitre cinq de Notre connaissance du monde extérieur de Russell (1914), intitulé La théorie de la continuité.

12 1930, p. 272. Que Brouwer estime important de ne pas s'écarter de l'idée classique de continuité, même si c'est bien sûr pour en donner un traitement moderne, on en trouvera un autre témoignage dans la façon dont il réagit à certaines idées de Weyl. Celui-ci avait écrit en 1921 que «!le concept de fonction continue dans un intervalle borné ne peut pas être défini sans inclure simultanément dans la définition le caractère continu et borné. [...] Ce qui est appelé de nos jours une fonction discontinue est constitué en réalité de plusieurs fonctions dans des continua séparés!»; et Brouwer notait en marge «!Très vrai, souligner, car c'est le point principal et le plus important!». Cf. P. Mancosu, From Brouwer to Hilbert, Oxford, Oxford University Press, 1998, p. 18.

${ }^{13}$ Les fondements de mathématiques (1927), dans Largeault, 1992, p. 154 (traduction légèrement modifiée). Ce passage figure déjà presque mot pour mot dans le texte Sur l'infini de 1925.
} 
devant la machinerie transfinie : c'est le moment où on quitte la terre ferme des mathématiques pour les créations verbales de la logique.

La solution de 1907. Assez tôt, les travaux de topologie de Brouwer sur la nature de la dimension l'avaient confirmé dans son sentiment qu'un abîme séparait les ensembles de points et le continu. En d'autres termes, la théorie des ensembles était inadéquate pour penser le continu : le rapport des points à la droite n'est pas un rapport d'appartenance tel qu'il est défini entre l'élément et l'ensemble ${ }^{14}$. De là le soin pris à distinguer systématiquement ensembles et continua.

Le principe des principes. Dès le début de sa carrière, Brouwer a vu dans l'idéalisme allemand le cadre approprié d'une bonne théorie du continu et encore en 1930 il estimera que «!la conception, qui fait du continu une intuition pure $a$ priori, dans le style de Kant et de Schopenhauer, se confirme pour l'essentiel à la lumière de l'intuitionnisme! ${ }^{15}$. Pour rendre compte de cette donnée première, il faudra introduire des objets mathématiques non-finis, inachevés ; quitter en d'autres termes le point de vue exclusivement statique adopté jusqu'alors en mathématique pour un point de vue dynamique.

Dès 1907, l'idée centrale est déjà là : le continu est une donnée première et il est vain de chercher à le construire à partir de points individualisés. Tout l'édifice des mathématiques repose sur une intuition originaire décrite comme!:

un substrat, privé de toute qualité, d'une perception quelconque du
changement, une unité du continu et du discret, la possibilité de penser
ensemble plusieurs entités reliées par un 'entre', laquelle n'est jamais
épuisée par l'insertion de nouvelles entités. Puisque le continu et le discret se
présentent comme des compléments inséparables, ayant tous deux des droits
égaux et étant tous deux également clairs, il est impossible d'éviter l'un
d'eux comme entité primitive, en essayant de construire l'un à partir de
l'autre.
L'intuition mathématique est incapable de créer autre chose que des
ensembles dénombrables d'individus. Mais elle est capable, après avoir créé
une échelle d'ordre $\square$, d'y surimposer un continu pris comme un tout, qui
peut réciproquement être pris par la suite comme un continu mesurable, qui
est la matrice des points sur l'échelle ${ }^{16}$.

Le continu mesurable : «!point set topology!» et pré intuitionnisme. Donné ainsi comme un tout, le continu est amorphe ; mais il peut servir de matériau sur lequel s'exerce l'activité constructive du mathématicien. Celui-ci doit commencer par le transformer en un continu mesurable, ce qu'il fait en définissant une échelle de type $\square$. Les points individualisés qui résultent de la construction de cette échelle restant dénombrables, la solution du problème du continu passe par la possibilité de définir une application d'un ensemble de points sur le continu. À cette fin, Brouwer commence par décrire les trois modes légitimes de formation d'ensemble de points sur le continu. L'étape suivante consiste alors à construire avec leur aide des ensembles de points, c'est-à-dire des sous-ensembles du continu. C'est l'examen de ces trois modes de

\footnotetext{
${ }^{14}$ Voir par exemple 1912, p. 46 : l'expression ensemble dont les éléments sont les points de l'espace «!est totalement dénuée de sens pour l'intuitionniste!».

${ }^{15}$ La structure du continu, p. 276.

${ }^{16} 1907$, respectivement p. 17 et 45 .
} 
construction qui permet d'apporter une solution au premier problème d'Hilbert : tous les ensembles définissables sur le continu sont soient dénombrables soient de la puissance du continu ${ }^{17}$.

Le raisonnement qui mène à cette conclusion est concis et peu clair. Comme ce passage fait partie de ce qui sera abandonné par la suite, il est permis de supposer que ce changement d'attitude tient à ce que rétrospectivement Brouwer lui-même le jugeât insatisfaisant. Ce qui est sûr en revanche, c'est que le point de vue adopté par Brouwer est très proche de celui des pré-intuitionnistes français : Borel, Lebesgue, Baire, partageaient l'attitude d'acceptation critique à l'égard des outils forgés par Cantor. Que l'on parle de topologie ensembliste ou de théorie descriptive des ensembles ${ }^{18}$, il s'agit d'aborder le problème du continu par en bas, de manière constructive, en construisant des ensembles de points de plus en plus grands. C'est d'ailleurs dans cette proximité qu'il faut chercher les raisons de l'insatisfaction rapidement manifestée par Brouwer : le résultat n'est pas faux, il est insatisfaisant; puisque tout ensemble dénombrable a une mesure de Lebesgue nulle, il est impossible, avec de telles méthodes, d'aller au-delà du continu réduit, et l'on reste donc sur le seuil du véritable problème. Dès cette époque, l'intuitionnisme se caractérise par le rôle déterminant joué par la prise en compte du connu. La difficulté à traiter du continu vient, nous dit-on, de ce que la plupart de ses éléments ne nous sont pas connus! ; il devient du même coup impossible de définir une application $^{19}$. Mais s'il en est ainsi, c'est qu'un rapport est établi entre connaissable et constructible : en règle générale, connaître un nombre et le construire ne sont qu'une seule et même opération ${ }^{20}$. Brouwer, si l'on préfère, associe étroitement individualisé et dénombrable. Reconnaître un objet, le distinguer des autres, c'est-à-dire encore l'individualiser, c'est être capable de lui donner un nom. Or, dire d'un ensemble qu'il est dénombrable c'est affirmer qu'à chacun de ses éléments, il est possible de faire de façon univoque un nombre, qui peut valoir comme nom dudit élément.

\section{LA CRITIQUE DES FONDEMENTS LOGIQUES DE LA THEORIE DES ENSEMBLES : LA THEORIE DES PUISSANCES ET LE PROBLEME DE LA SECONDE CLASSE DE NOMBRES}

Au contraire de ce premier aspect de la dissertation de 1907 appelé - en tant qu'insatisfaisant - à disparaître d'un intuitionnisme en pleine possession de ses moyens, et sur lequel il n'est donc pas nécessaire de s'étendre, le refus de la seconde classe de nombres, et la critique des fondements logiques de la théorie des ensembles qui lui sert de toile de fond, représentent, eux, un acquis définitif, sur lequel Brouwer ne reviendra

\footnotetext{
${ }^{17}$ Cf. la discussion, beaucoup plus détaillée, de l'hypothèse du continu, des positions respectivement défendues par les deux parties, dans Intuitionnisme et formalisme. Ce texte de 1912 a profité de l'examen critique de la première édition de l'ouvrage de Schoenflies, Entwickelung der Mengenlehre und ihrer Anwendungen (Leipzig, Teubner, 1908), qui a permis à Brouwer de préciser son point de vue et marque à ce titre une étape importante dans son évolution. Voir à ce propos l'ouvrage de van Dalen cité note 3, en particulier p. 208 sqq.

${ }^{18}$ Dès 1938, Cavaillès parlait de «!théorie concrète des ensembles!» (Méthode Axiomatique et formalisme, Paris, Hermann, 1938, p. 6).

${ }^{19} 1907$, p. 84!; cf. encore p. 86, l'objection contre les définitions créatrices. C'est en cela que Poincaré concède trop à Hilbert!: il ne suffit pas de définir, même si la définition est consistante, il faut s'assurer que l'objet correspondant existe.

${ }^{20}$ Par exemple, p. 81 : «!...quelque chose qui ne peut être pensé, c'est-à-dire qui ne peut être mathématiquement construit!».
} 
plus : «!La théorie des puissances (...) illustre fort clairement l'existence de l'abîme infranchissable qui sépare les deux écoles!» ${ }^{21}$.

Si l'opposition apparaît cette fois irréductible, c'est que la création cantorienne y est abordée sous un autre aspect. L'examen se déroule dans un nouveau contexte, celui du chapitre trois, intitulé Mathématique et logique. L'erreur des cantoriens y est rapprochée de celle des axiomaticiens, accusés de surajouter aux constructions mathématiques des structures logiques qui leur sont étrangères. Plus précisément, il est reproché au fondateur de la théorie des ensembles d'emprunter aux logiciens un mode de formation d'ensembles à quoi rien ne correspond dans la pratique des mathématiciens.

La question est de savoir comment engendrer la suite des alephs. Chez Cantor, deux méthodes sont autorisées : l'exponentiation, puisque $2^{\square \mathrm{n}}>\square_{\mathrm{n}}$; mais aussi le passage par les nombres de la seconde classe. Brouwer, pour sa part, n'autorise que l'itération ; transfinie, sans doute, mais uniquement pour des éléments égaux, ce que Cantor ne respecte pas. Comme précédemment, on présentera dans un premier temps la création cantorienne, avant d'examiner les objections intuitionnistes, et leur contrecoup sur la question du continu.

Cantor. On a vu que, pour créer de nouveaux cardinaux, la théorie des ensembles pouvait utiliser l'exponentiation transfinie. Brouwer, comme d'ailleurs Borel, avait émis des doutes sur la possibilité d'effectuer cette opération. La question se pose donc : n'y aurait-il pas d'autres procédures permettant de construire des puissances sans cesse plus grandes ? Cantor avait répondu par l'affirmative et la première définition de $\square_{1}$ utilisait une méthode beaucoup plus rapide, qui en faisait la puissance de la seconde classe de nombres. À quoi ses adversaires objecteront : c'est encore pire. Alors que, dans le premier cas il ne s'agissait que de réserves, cette fois, le non! est catégorique.

Pour comprendre cette nouvelle approche, il convient de partir cette fois de la théorie des nombres ordinaux. Une fois donné le premier nombre ordinal, 0 , qui est par définition l'ensemble vide, les nombres ordinaux sont engendrés par l'application de l'un ou l'autre de ces deux principes :

(I) donné un ordinal $a$, former le plus petit des ordinaux plus grands que $a$, noté $a+1$;

(II) donnée une suite croissante $a_{\mathrm{n}}$ d'ordinaux, former le plus petit des ordinaux plus grands que tous les $a_{\mathrm{n}}$, noté $\lim \left(a_{\mathrm{n}}\right)^{22}$.

Les ordinaux finis sont engendrés à l'aide du seul premier principe. Ils forment ce que Cantor a appelé la première classe de nombres, dont la puissance est infinie dénombrable. La seconde classe de nombres est alors définie!:

comme l'ensemble de tous les nombres successifs formulables à l'aide de ces deux principes d'engendrement (ou encore de tous les ordinaux infinis dénombrables), où (II) est appliqué seulement aux suites dénombrables $\left\{a_{n}\right\}$.

De même que par définition $\square_{0}$ est la puissance de la première classe de nombres, $\square_{1}$ est, par définition, la puissance de la seconde classe de nombres. Aucun des nombres

\footnotetext{
${ }^{21} 1912$, p. 48.

${ }^{22}$ Ces ordinaux limites sont parfois encore appelés nombres de la seconde espèce, ce qui peut créer une fâcheuse confusion avec les nombres de la seconde classe.
} 
de la seconde classe n'a pour puissance $\square_{1}$, ce qui revient à dire qu' $\square_{1}$ n'est pas dénombrable, qu'en un sens il n'est pas accessible «!d'en bas!».

La position de Brouwer : le cour de l'argument. Si Brouwer et les préintuitionnistes étaient réservés devant l'exponentiation transfinie, cette fois leur refus est encore plus énergique. Autant chacun des deux principes susmentionnés est légitime, autant la seconde classe de nombres n'existe pas. À première vue, un tel refus a de quoi surprendre : comment accorder les deux principes d'engendrement sans accorder l'existence d'une classe composée d'éléments engendrés à partir des dits principes. Qu'on la juge ou non inutilement subtile, la réponse dit ceci : quand il parle de l'ensemble de tous les ..., «!Cantor mentionne quelque chose qui ne peut être pensé, c'est-à-dire qui ne peut pas être mathématiquement construit ; car une totalité construite au moyen de 'etc.' ne peut être pensée que si le 'etc.' réfère à un type d'ordre $\square$ d'objets égaux, mais cet etc. ne réfère ni à un type d'ordre $\square$, ni à des objets égaux. Ici Cantor perd le contact avec la terre ferme des mathématiques!» ${ }^{23}$. Une page plus haut, passant en revue les seuls modes de construction d'ensemble légitimes du point de vue intuitionniste, Brouwer écrivait encore «!nous ne pouvons créer que des séquences finies, puis le type d'ordre $\square$, au moyen du clairement conçu 'etc.', mais seulement consistant d'éléments égaux ; [...] et finalement le continu intuitif!» ${ }^{24}$.

Un exposé exhaustif demanderait d'examiner pas à pas la construction de la suite transfinie des ordinaux, et la forme normale de Cantor qui la résume. Il faudrait, en d'autres termes, regarder de plus près à quoi peut ressembler cette application répétée des deux principes, et ce qui en résulte; ce qui nous entraînerait trop loin. On se limitera donc aux quelques remarques suivantes. Le bon etc. s'en tient à la répétition de l'identique. Or ce n'est pas le cas ici : la suite qui décrit le résultat de l'application des deux principes contient plusieurs etc. (ou, pour parler comme Borel, «!une infinité énumérable de passages à la limite successifs ou superposés!») qui ne respectent pas les conditions requises pour que l'usage en soit légitime et sur lesquelles il convient peutêtre de revenir : pourquoi exiger que le etc. ne s'applique qu'à des éléments égaux!? Dans le passage cité à l'instant, Brouwer ajoutait en effet en note : «!l'expression etc. signifie la répétition indéfinie d'un seul et même objet ou opération, quel que soit le degré de complexité de la définition de l'objet ou de l'opération!». Dans le même ordre d'idée, Borel remarquait que, s'il est vrai que «!ce n'est pas en soi la même chose d'ajouter 1 à 1 pour obtenir 2, ou d'ajouter 1 à 2 pour obtenir 3 [...] l'opération par laquelle l'addition d'une unité permet avec chaque entier de former l'entier suivant peut être indéfiniment considérée comme étant, à un certain point de vue, la même!»» ${ }^{25}$. Quand on quitte le domaine des entiers, on ne peut plus en dire autant, et c'est pourquoi la notion de seconde classe de nombre n'est pas claire.

\footnotetext{
${ }^{23}$ Op. cit., p. 81.

${ }^{24}$ Op. cit., p. 80. Il semble y avoir par moment un certain flottement dans la terminologie de Brouwer : tantôt, comme ici, nous pouvons créer le continu, tantôt il nous est donné et nous pouvons seulement le concevoir.

${ }^{25}$ E. Borel, Leçons sur la théorie des fonctions, Gauthier-Villars, 1950 (4e édition), p. 145 ; le passage est extrait d'un texte intitulé l'antinomie du transfini, initialement publié en 1900 dans la Revue philosophique ; cf. encore p. 227-228 : «!Un nombre transfini de la seconde classe n'est pas autre chose qu'une notation abrégée, pour indiquer l'ordre dans lequel doivent être effectuées une infinité énumérable d'opérations, comportant une infinité énumérable de passages à la limite successifs ou superposés!»; ou p. 160 : «!Il n'est donc pas possible qu'on arrive à une notation bien définie pour l'ensemble des nombres que Cantor appelle nombres de la seconde classe!». J. Bouveresse signale une parenté entre la position de Borel et celle de Wittgenstein, La force de la règle, Paris, Minuit, 1987, p. 37-38.
} 
Logique et mathématique. Que reste-t-il alors!? D'un point de vue strictement intuitionniste, la position de Cantor n'a donc pas de sens. Il est tentant d'établir un parallèle avec Russell, la théorie des types reposant elle aussi sur un partage établi entre sens et non-sens!; mais pour ce faire, le philosophe anglais cherche la solution du côté de la logique, du langage : il énonce des règles de syntaxe, ce qui est aux antipodes du point de vue adopté par Brouwer, qui s'oriente plutôt dans la voie suivie par Husserl : une théorie des actes, de la visée, du remplissement. Par exemple, est-il remarqué p. 82, bien que le dénombrablement inachevé n'existe pas comme totalité, on peut cependant l'admettre comme intention ; mais il n'y a pas de remplissement ${ }^{26}$. Ce n'est pas un hasard si $\mathrm{H}$. Weyl, qui a le plus souligné ce rapport de l'intuitionnisme et de la phénoménologie, a également insisté sur la spécificité de la pratique mathématique : la mathématique est plus un faire qu'un savoir : ein Tun, nicht eine Lehre $e^{27}$. Le même Weyl a lui aussi fortement souligné la dépendance de la théorie des ensembles à l'égard de la logique. En un sens, l'idée n'est guère originale. La thèse logiciste par exemple présuppose l'idée d'une grande logique qui inclut, sinon la totalité, du moins une partie de la création cantorienne. Fonder les mathématiques sur la logique, c'est en fait la fonder sur la théorie des ensembles, sans quoi on s'expliquerait mal comment les paradoxes surgis au sein de cette dernière ont pu provoquer une crise des fondements. Mais il s'agit cette fois non pas de présenter la théorie des ensembles comme «!une discipline extrêmement voisine de la logique pure!» mais de souligner «!le caractère intenable des positions logiques d'où est partie la méthode ensembliste!» ${ }^{28}$. L'intuitionniste n'accepte pas sans réserve le partage habituellement fait entre théorie des ensembles d'un côté et logique de l'autre, lequel permet de tenir la première pour seule responsable des contradictions et de mettre hors de cause la seconde. En réalité, avec l'apparition de domaines infinis, c'est toute la théorie de la quantification qui demande à être revue ${ }^{29}$.

On évitera donc de parler de nombre de la seconde classe, car c'est laisser entendre qu'on admet l'existence de cette seconde classe. Pour faire sens du raisonnement cantorien, il faut le modifier légèrement, en l'occurrence le transposer du domaine des mathématiques à celui de la logique, i. e., Brouwer étant kantien, du synthétique à l'analytique. Quand on sait le peu de cas que le mathématicien hollandais faisait de la discipline fondée par Aristote, on ne s'étonnera pas qu'il ne trouve plus au bout du compte que la trivialité suivante :

\footnotetext{
${ }^{26}$ Sur Brouwer et la phénoménologie, on se reportera désormais à la dissertation de Mark van Atten, Phenomenology of choice sequences, Utrecht, 1999.

${ }^{27}$ Cf. J. Largeault, Intuition et intuitionisme, Paris, Vrin, 1993, p. 82-83, qui rappelle que la construction est conçue comme une expérience, ce qui enveloppe la critique de l'a priori et parle en faveur du point de vue empirique en mathématique, dans la ligne des idées développées par Paul DuBois Reymond dans sa théorie générale des fonctions de 1882.

${ }^{28}$ Respectivement dans Sur les définitions des concepts mathématiques fondamentaux (1910) et Les degrés de l'infini (1931), tous deux traduits par Jean Largeault dans : H.Weyl, Le continu et autres écrits, Paris, Vrin, 1994, p. 27 et 303.

${ }^{29}$ «!On doit comprendre d'une façon hypothétique l'énoncé qui porte sur 'chaque nombre', comme l'énoncé qui exprime quelque chose seulement sous la condition qu'un nombre est donné réellement; et en conséquence il n'est pas susceptible d'être nié. L'assertion d'existence n'acquiert de contenu que par l'exhibition d'un exemple : ce nombre déterminé, construit de telle manière, a la propriété P. L'absolutisme existentiel saute par dessus ce genre de scrupules fondés sur la nature de l'infini ; il prend ces énoncés comme des énoncés ordinaires susceptibles de négation et qui peuvent être opposés dans le tertium non datur [...] on a traité un champ de possibilités constructives comme un champ fermé existant en soi!», Les degrés de l'infini, cité à la note précédente, p. 301-302.
} 
Si l'on admet l'entité logique T (puissance de la seconde classe de nombre), alors l'axiome $T=A$ (A étant la puissance de $\square$ ) engendrera une contradiction. De même pour l'entité logique I censée désigner un nombre cardinal vérifiant l'inégalité $A<I<T$.

Le contenu mathématique de ces affirmations se réduit à ceci :

$\left.1^{\circ}\right)$ Il est faux que la seconde classe de nombres soit concevable et dénombrable ;

$2^{\circ}$ ) Il est faux que la seconde classe de nombres soit concevable et qu'on puisse trouver un cardinal dont la puissance soit comprise entre celles de la seconde et de la première classe de nombres.

Mais cela, l'intuitionniste le savait déjà : pour qu'une conjonction soit fausse, il faut et il suffit que l'un de ses membres le soit ; or, ce que l'intuitionniste conteste aux cantoriens, c'est précisément la possibilité de concevoir effectivement la seconde classe de nombres. On retrouve bien ici ce leitmotiv de la critique de Brouwer : toute une partie de la théorie des ensembles relève en réalité de la logique et, à ce titre, n'a pas sa place en mathématique.

De la même façon, on peut accepter de parler de l'ensemble de tous les ordinaux et de sa puissance. Mais pour cela, il faut introduire un nouveau concept, celui de dénombrablement inachevé, qui se situe entre l'infini dénombrable et le continu. Un ensemble dénombrablement inachevé $E$ satisfait a deux conditions :

a) on ne peut jamais en construire de façon bien définie qu'un sous-ensemble dénombrable $S$;

b) donné l'un quelconque de ces sous-ensembles, on peut toujours en déduire, par un procédé défini à l'avance, par exemple la diagonalisation, de nouveaux éléments de $E$ n'appartenant pas à $S$.

On dira alors que l'ensemble des nombres ordinaux, ou encore l'ensemble des points définissables sur le continu, sont dénombrablement inachevés. Mais il faut s'empresser d'ajouter que stricto sensu, $E$ n'existe pas comme un tout déjà déployé devant le savant, et que sa puissance, elle, n'existe pas du tout. On aura reconnu, dans cet inachevé, le point de vue dynamique si caractéristique de l'intuitionnisme, qui introduit le devenir dans le monde jusqu'alors statique des mathématiques. Croire que la position de Brouwer enveloppe le rejet de l'infini en acte serait donc une grave erreur. Refuser la seconde classe de nombres, ce n'est pas du tout refuser l'infini actuel. Porter, comme le fait Poincaré, la critique sur ce dernier point, c'est rester sur le seuil du problème, «!qui gît plus profond, nous dit la conclusion de l'ouvrage, à savoir dans la confusion entre l'acte de construire les mathématiques, et le langage des mathématique!». Sur cette question, le mathématicien hollandais prend parti contre son aîné et se range résolument aux côtés de Cantor : «!Quant à l'infini actuel des cantoriens, il existe bien, pourvu que nous le confinions à ce qui peut être intuitivement construit, et que nous nous abstenions de l'étendre par des combinaisons logiques qui ne peuvent pas être réalisées!» ${ }^{30}$.

\footnotetext{
${ }^{30}$ Op. Cit., p. 97.
} 


\title{
Les contrecoups sur l'hypothèse du continu
}

- directs!: l'introduction de la seconde classe de nombre permet, on l'a vu, de reformuler l'hypothèse du continu, qui devient alors (p. 83) :

La totalité des nombres de la seconde classe et la totalité des points du continu ont même puissance.

Dès lors que l'on refuse toute existence mathématique aux deux concepts figurant dans cette affirmation, il n'en reste plus qu'un théorème logique, qui s'énonce ainsi :

\begin{abstract}
Il n'est pas contradictoire de supposer, entre la totalité des nombres de la seconde classe et la totalité des points du continu, entendues comme entités logiques, une correspondance bi univoque qui ne laisse de côté aucun élément des deux totalités.
\end{abstract}

Pour démontrer ce théorème, il suffit de remarquer que, une fois abandonnée l'intuition du continu au profit d'une totalité des points du continu, ceux-ci demandent à être définis, ce qui ne peut être fait qu'en donnant des lois pour le développement des fractions binaires qui les approchent. En ce sens, le continu est dénombrablement inachevé, ce qui, on l'a vu, est aussi le cas de la totalité des nombres de la seconde classe $^{31}$.

- Indirects!: l'article de 1912 permet de compléter ces indications. Cette fois, ce n'est pas moins de trois lectures qui nous sont proposées, et Brouwer prend soin de préciser dans chaque cas les points d'accord et de désaccord entre lui et son adversaire.

1) Entendue comme affirmant l'impossibilité de construire sur l'intervalle unité des ensembles infinis de réels dont la puissance serait plus grande qu' $\square_{0}$ mais plus petite que celle du continu, l'hypothèse du continu est vraie, comme l'a démontré le premier chapitre de la dissertation.

2) Entendue comme affirmant la possibilité d'établir une correspondance bi univoque entre les éléments d'un ensemble infini dénombrable de nombres ordinaux d'une part et ceux d'un ensemble de réels définis sur l'intervalle unité de l'autre, et cela de telle sorte que la correspondance subsiste, quelle que soit la façon de prolonger la construction des deux ensembles - entendue ainsi, l'hypothèse du continu est également vraie.

3) En revanche elle cesse de l'être quand elle affirme la possibilité de construire une loi associant d'une part un nombre ordinal infini dénombrable à chaque série élémentaire de chiffres, et garantissant d'autre part que deux séries élémentaires distinctes ne seront jamais associées au même ordinal. La puissance de l'ensemble des ordinaux concernés par la loi ne peut en effet dépasser $\square 0^{32}$.

\footnotetext{
${ }^{31}$ Borel pointe dans la même direction lorsqu'il rappelle que : a) s'il est vrai que, quelque grand que soit le nombre transfini $X$ que l'on sera arrivé à définir, l'ensemble des nombres transfinis inférieurs à $X$ est dénombrable, il ne s'ensuit pas que : b) l'ensemble des nombres de la seconde classe soit dénombrable ; cf. Les paradoxes de l'infini, Paris, Gallimard, p. 111.

32 1912, p. 50-51.
} 


\section{CONCLUSION}

La Dissertation de 1907 est donc bien une œuvre de percée. Elle contient des résultats importants, aussi bien mathématiques que philosophiques : la validité de l'intuition kantienne, le rapport (ou plus exactement l'absence de rapport) entre mathématique et logique et la mise hors circuit du langage qui en résulte, la théorie des constructions. Certes le plus original, les véritables mathématiques intuitionnistes (suites de choix, grands théorèmes de la barre ou de l'éventail) manquent encore ; mais il ne faut pas perdre de vue que ces créations sont nées d'une réflexion critique sur la théorie des ensembles : pourquoi cela ne marche-t-il pas ? sur quels principes s'appuyer pour avoir une bonne théorie du continu?

Une attitude nuancée. Contrairement à ce qu'on croit parfois, le refus dans ce domaine n'est pas total ; au point de départ, il y a plutôt une admiration, presque une gratitude devant les nouveaux domaines conquis par Cantor pour les mathématiques. Pour cette raison, il ne s'agit pas tant de rejeter en bloc la théorie des ensembles que de la réformer!; d'où les équivoques liées à l'emploi d'une terminologie détournée de son sens usuel, et les obstacles en partie inutiles rencontrés par les contemporains de Brouwer.

Décrit à grands traits, ce travail de discrimination laisse apercevoir deux grandes lignes de partage. Entre mathématique et logique tout d'abord. Si Cantor s'est trompé, c'est qu'il a introduit en mathématiques des considérations logiques qui n'y ont pas de place. La crise des fondements appelle le diagnostic suivant : logique et mathématiques sont comme partie malade et partie saine. Le remède consistera donc à empêcher la contamination de celle-ci par celle-là. Il prend également la forme d'un processus de traduction visant à reformuler, dans la mesure du possible, en termes mathématiques authentiques les affirmations ensemblistes où figurent des concepts logiques ${ }^{33}$.

Toutefois, même au plan mathématique, les apports de Cantor ne sont pas tous à conserver. Dans la création cantorienne, la critique intuitionniste vise avant tout la théorie des cardinaux (cf. la citation de 1912 supra) beaucoup plus que la théorie des ordinaux, qui, si elle n'est pas entièrement conservée (il faut que les ordinaux soient constructibles !) est considérée comme un apport durable de Cantor à la mathématique. En particulier, il y a lieu de souligner que, pour autant qu'on ne va pas au delà des ordinaux constructibles, l'induction transfinie est légitime du point de vue intuitionniste, ce qui veut dire notamment que celui-ci est en un sens beaucoup plus libéral que le finitisme de Hilbert, ce que Gentzen ou Gödel ont très vite compris. On se rappellera également que la théorie des cardinaux repose sur la théorie des ordinaux : les cardinaux ne sont qu'une variété d'ordinaux ; de plus, dans la hiérarchie des alephs, les indices sont des ordinaux.

L'hypothèse du continu. Parmi les mérites des mathématiques cantoriennes, le moindre n'est pas d'avoir vu la nécessité de disposer d'une bonne théorie du continu, mais la confusion dénoncée à l'instant entre logique et mathématique a réduit à peu près à néant la contribution du point de vue ensembliste dans ce domaine. La conjecture communément appelée hypothèse du continu n'a pas une mais plusieurs formulations. Mathématiquement parlant, deux cas se présentent : stricto sensu, elle n'a pas de sens ;

\footnotetext{
${ }^{33}$ Cela signifie aussi qu'à côté de la traduction proprement mathématique, il en existe une autre dans le langage de la logique ; encore une fois, le défaut majeur et rédhibitoire de Cantor est d'être tombé dans la confusion des genres.
} 
lato sensu, elle est tantôt vraie, tantôt fausse ; par ailleurs, il est possible d'en donner des traductions logiques qui ne sont le plus souvent que des trivialités, ce qui n'est pas surprenant puisqu'on est passé du synthétique à l'analytique. Au cœur de la critique se trouve le concept de seconde classe de nombres, dont on a vu qu'elle est irrecevable parce que, contrairement à ce qui se passe dans le cas des nombres naturels, l'itération ne s'applique pas à des éléments égaux.

Les années 1907-1908 resteront comme des années fastes dans l'histoire des mathématiques, et il est regrettable que la dissertation de Brouwer soit le moins lu des trois textes (les deux autres étant dus à Zermelo et à Russell) où ont été alors formulées les grandes théories qui continuent aujourd'hui encore à dominer la problématique des fondements. 